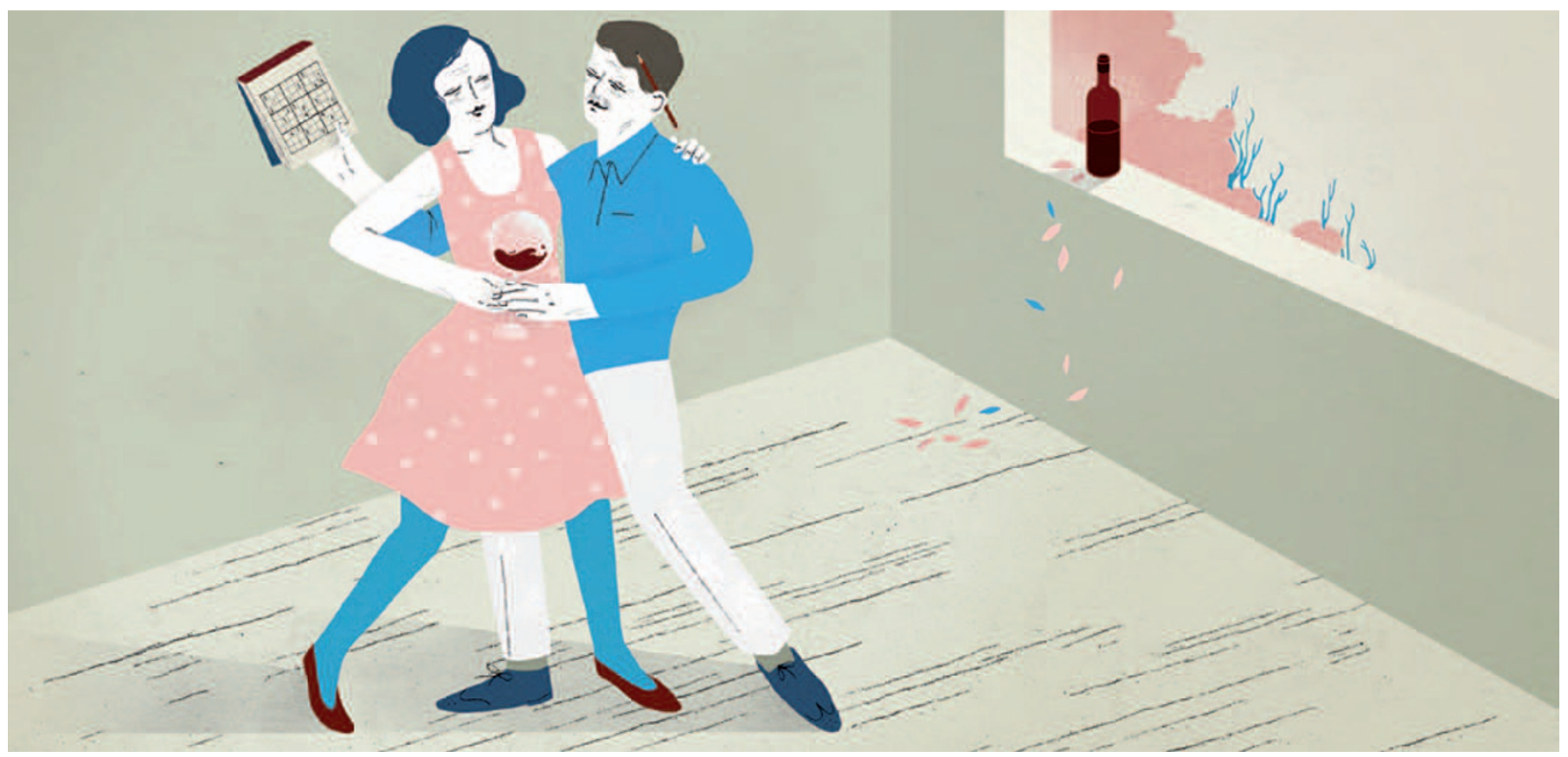

\title{
Activity is the best medicine
}

\section{Can exercise, social interaction and the Mediterranean diet really help to keep the cognitive decline of Alzheimer's disease at bay?}

\section{BY SARAH DEWEERDT}

$\mathrm{R}$ humba. Lindy hop. Cha-cha. Ballroom dancing may not be the first preventive treatment for Alzheimer's disease that springs to mind, but it is an ideal prescription for those concerned about their declining memory. In fact, says Perminder Sachdev, a neuropsychiatrist at the University of New South Wales in Sydney, Australia, dancing has a perfect blend of elements that help stave off dementia. "There's cognitive activity, there's also physical activity, and there's social interaction as well."

A healthy Mediterranean-style diet is also thought to be protective - so that dance class could be topped off with a big Greek salad and a glass of red wine.

Over the past decade, epidemiological studies have shown that exercise, intellectual activity, social relationships and a healthy diet all lead to a lower risk of dementia. Such findings have to be interpreted with caution, however, because many researchers are sceptical about

D NATURE.COM more research on the links between lifestyle and dementia go.nature.com/ivedp8
Even so, "we have enough suggestive observational data now from several studies" to conclude that lifestyle factors are important in Alzheimer's disease, much as they are in cardiovascular disease, says Ronald Petersen, director of the Alzheimer's Disease Research Center at the Mayo Clinic in Rochester, Minnesota.

\section{FIT AND HEALTHY}

The task now is to move from lifestyle factors to interventions - to find out how much exercise, what kind of intellectual activity and at what stage each could influence the course of the disease. "We need to do more [clinical trials] where we actually intervene" with cognitive activity, training programmes and exercise, and with an appropriate control group, Petersen says.

Some of these trials are already under way. For example, in the Fitness for the Aging Brain Study, researchers ${ }^{1}$ in Australia recruited 170 people who were worried that their memory had deteriorated or who had mild cognitive impairment (MCI), a condition that is considered a precursor to Alzheimer's disease. The researchers assigned half of the participants to a six-month exercise programme, either walking or doing other aerobic exercise for 50 minutes, three times a week. The other half, in the control group, carried on with their usual level of activity.
After six months, those in the exercise group slightly improved their scores on the cognitive section of the Alzheimer's Disease Assessment Scale (ADAS-Cog), a series of short memory, language and reasoning tests, whereas control subjects declined at a rate consistent with normal ageing. What's more, the exercise had lasting effects, leading to better scores 12 months after the programme ended.

ADAS-Cog is commonly used in clinical trials of Alzheimer's disease drugs, so the researchers were able to compare the effects of exercise with those of drugs called acetylcholinesterase inhibitors, which reduce the breakdown of the neurotransmitter acetylcholine. For people with MCI, regular exercise "can help your brain more than taking the medication that is currently available for Alzheimer's disease", says one of the researchers, Nicola Lautenschlager, who studies geriatric psychiatry at the University of Melbourne.

\section{PHYSICAL CHANGES}

How does this connection between body and mind work? Studies in rodents have suggested at least two different mechanisms ${ }^{2}$. First, exercise increases the activity of an enzyme called neprilysin that metabolizes amyloid- $\beta$ - the protein that makes up the characteristic plaques of Alzheimer's disease - and might 
help clear it from the brain. Physical activity also turns on the production of brain chemicals such as nerve growth factors, which promote the formation of nerve cells and the connections between them. This process is thought to make the brain better able to cope despite the pathological changes of Alzheimer's disease.

In the past few years, the development of biomarkers (see 'Warning signs', page S5) that can indicate Alzheimer's-related brain changes in living people have allowed researchers to explore more fully the mechanisms of the mind-body connection. For example, one study this year of 120 sedentary but healthy older adults without any memory problems assigned half the participants to a 3-days-a-week programme of physical exercise ${ }^{3}$. After a year, researchers performed magnetic resonance imaging (MRI) on several brain areas, including the hippocampus, the brain structure responsible for memory formation.

In older adults, the hippocampus typically shrinks by $1-2 \%$ each year, and this is what happened in the control group. But in the exercise group, the volume of the hippocampus actually increased by $2 \%$. "That's probably millions of cells," says research team member Kirk Erickson, a psychologist at the University of Pittsburgh, Pennsylvania. With one year of exercise, "we are in essence rolling back the clock by one to two years".

\section{BRAIN TRAINING}

Another report ${ }^{4}$, also published this year, suggests that similar mechanisms are at work when people exercise their brains. Canadian researchers used functional MRI to analyse brain activity in 15 people with MCI. After a one-week programme designed to teach the participants new memory strategies, there was activation in several additional brain regions during memory tests, suggesting that intact areas of the brain were able to take over from damaged areas. The participants also scored better on the tests.

Many studies of cognitive stimulation and dementia make use of computer games designed to boost mental skills. Although such 'brain training' interventions do not generally make healthy people smarter, they produce positive results in people with Alzheimer's disease and related conditions. One 2006 trial funded by the US National Institutes of Health showed that brain training can counteract some of the cognitive decline expected with ageing $^{5}$. In that study - known as Advanced Cognitive Training for Independent and Vital Elderly (ACTIVE) - people over 65 years of age who did a five- to six-week brain training programme focusing on memory, reasoning or speed of processing skills were better at these skills than control participants even five years later.

Computerized brain training programs are popular among researchers because these interventions are controllable and predictable, especially compared with intellectual pursuits in the real world. But this doesn't mean that people need to play computer games to stay mentally agile, says Sachdev. Instead, he argues, people are likely to benefit from any intellectual pursuit that both requires effort ("something where you challenge your brain") and is enjoyable ("so that you can sustain it"). That could mean anything from taking up the clarinet to doing Sudoku puzzles.

\section{FOOD FOR THOUGHT}

Meanwhile, other lifestyle factors that can modify the risk of Alzheimer's disease are continuing to emerge through epidemiological research. These types of studies, involving observation of thousands of people and their habits, underpin our knowledge about the Mediterranean diet, which includes a relatively high consumption of fruits, vegetables, whole grains and olive oil, relatively low consumption of red meat and saturated fat, and a glass of red wine with dinner.

Eating these foods has already been shown to reduce the risk of cardiovascular disease, hypertension and diabetes. In the past few years, three independent epidemiological studies conducted in New York $^{6}$, Chicago ${ }^{7}$ and Bordeaux, France ${ }^{8}$, have shown that those who eat mostly Greek peasant food also stay the sharpest mentally. "There has been converging evidence that adherence to such a diet is related to lower risk of cognitive decline or Alzheimer's disease," says Nikolaos Scarmeas, a neurologist at Columbia University in New York.

A team of Columbia University researchers including Scarmeas asked 1,880 New Yorkers detailed questions about their eating habits, then studied them for an average of five-anda-half years. They found that the people with the most Mediterranean diet have up to a $40 \%$ lower risk of developing Alzheimer's disease than those who eat less Mediterranean food ${ }^{6}$. Results like these are so promising that several groups around the world are planning randomized trials of the Mediterranean diet as a way of preventing Alzheimer's disease.

Evidence that social engagement helps to prevent dementia also comes primarily from observational studies. For example, among more than 6,000 people aged 65 or older in Chicago, those with the most extensive social networks and the highest levels of social engagement have the lowest rates of cognitive decline ${ }^{9}$.

It can be difficult to measure people's level of social engagement and it is even harder to design randomized trials to investigate it. Disentangling the effects of social engagement from those of other lifestyle elements is far from straightforward. Still, social engagement is a form of intellectual engagement, argues Linda Teri, professor of psychosocial and community health at the University of Washington in Seattle. Teri has designed programmes to encourage physical activity and social connections in people with MCI and dementia. "When we are with other people, we are listening to the conversation, we're tracking ideas, we're forming our own ideas," she says. "We're actually engaging in quite a bit of cognitive skills."

So people who exercise in groups may benefit from both the social stimulation and the physical activity. For example, consider Erickson and colleagues' research into exercise and brain changes in healthy older people ${ }^{3}$. Instead of aerobic exercise, the control group met three times a week to do stretches. This did not increase the size of their hippocampus, but it did improve their scores on a simple computerized test of memory, similar to the improvements in the exercise group. Erickson suggests that this social stimulation benefits other parts of the brain that the study did not measure.

\section{A LITTLE BIT BETTER}

In some parts of the research community, the argument that lifestyle can help to delay Alzheimer's disease is a tough sell. Last year, the US National Institutes of Health organized a consensus panel on preventing Alzheimer's disease. It concluded that it is too soon to tell whether lifestyle changes - or any other prevention strategy - can affect the development or the course of Alzheimer's disease.

Even those who are more bullish about the evidence say that lifestyle changes are likely to have only a limited benefit. But because Alzheimer's disease develops late in life, even small changes in risk or slight delays in the development of symptoms could greatly reduce the burden of disease, as people would be more likely to die from other causes before becoming mentally impaired.

As Erickson says: "If we can at least prevent some of the normal age-related decline from happening, even if it doesn't eliminate the risk - if it just reduces the risk of developing Alzheimer's disease or makes the quality of life a little bit better - I think we've gone a long way."

Sarah DeWeerdt is a science writer based in Seattle, Washington.

1. Lautenschlager, N. T. et al. J. Am. Med. Assoc. 300, 1027-1037 (2008).

2. Savica, R. \& Petersen, R. C. Psychiatr. Clin. N. Am. 34, 127-145 (2011).

3. Erickson, K. I. et al. Proc. Natl Acad. Sci. USA 108, 3017-3022 (2011)

4. Belleville, S. et al. Brain 134, 1623-1634 (2011).

5. Willis, S. L. et al. J. Am. Med. Assoc. 296, 28052814 (2006).

6. Scarmeas, N. et al. J. Am. Med. Assoc. 302, 627-637 (2009).

7. Tangney, C. C. et al. Am. J. Clin. Nutr. 93, 601-607 (2011).

8. Féart, C. et al. J. Am. Med. Assoc. 302, 638-648 (2009).

9. Barnes, L. L., Mendes de Leon, C. F., Wilson, R. S., Bienias, J. L. \& Evans, D. A. Neurology 63, 2322-2326 (2004) 